\title{
Impact of Using Java Software of Geometrical Optics JSGO on the Construction Success of the Virtual Image among the First Year University Students
}

\author{
${ }^{1}$ Abderrahmane Ouazzani Touhami, ${ }^{2}$ Nadia Benjelloun, ${ }^{3}$ Mohammed Alami and \\ ${ }^{4}$ Haddou Aouni \\ ${ }^{1,2,3,4}$ Interdisciplinary Laboratory of Research in Didactics of Sciences and Technology (ILRDST), Faculty of \\ sciences Dhar Mehraz, Sidi Mohammed Ben Abdellah University, PB: 1796-30000, Fez, Morocco
}

\begin{abstract}
This paper presents a study of the impact of using Java Software of Geometrical Optics JSGO on the geometrical construction success of the virtual image among the first year university students, option: Sciences of Physical Matter SPMThe experimentation realized was conducted towards the end of the university year 2013/2014 among two groups of learners consisting each of them of 59 students: a witness group and another experimental. The witness group must to use only the traditional method, paper, pencil, ruler and protractor, for doing tests; while the experimental group should construct the virtual image using only the mentioned software. We have scored that using JSGO has no significant influence on the success of the geometrical construction of the virtual image associated with the first variant sub-tests. However, using this tool through the second variant sub-tests leads to a number of correct constructions which is higher than that noticed when using traditional method.By analyzing the results concerning "students' conceptual difficulties" we have scored the presence of the conceptual difficulty associated with an incorrect status that about $32 \%$ of witness group students attribute to the construction ray extension. The use of JSGO provides a useful tool to overcome this conceptual difficulty. This analysis also indicates that, despite the interactive character of JSGO, about $9 \%$ of experimental group students still face the conceptual difficulty related to the correspondence image object (punctual and global) and also to the conceptual difficulty related to the minimum number of rays departing from the same point of the luminous object.Finally, the study of "students" perceptions about using JSGO" showed that the participants are very satisfied with using this computer wizard.
\end{abstract}

Keywords: Java Software of Geometrical Optics JSGO; virtual image; witness group; experimental group.

\section{Introduction And Problematic}

In the first instance, many researchers have shown that the integration of computer technology in the geometrical optics teaching has a positive influence on the acquisition of certain concepts. Bayrak (2008) investigated the effectiveness of the teaching assisted by computer in relation to the traditional teaching. The experimental group consisted of biology students who received a teaching of geometrical optics by using the simulation, whereas the control group consisted of chemistry students who received the same teaching by the traditional method. The results showed that the control group was less successful than the experimental group.

(Ahaji and al, 2008) analyzed the effect of integrating a simulation of geometrical optics on the pupils' learning in the first year of Baccalaureate, option: experimental sciences. The results showed that the integration of this simulation has a positive effect on the teaching process.

In a second instance, the results presented by other researchers showed that it is not easy to prove the benefits of the integrating of the software tools on students' teaching.

Clark (2011) examined the teaching of image formation using converging, diverging lenses and mirrors among the students. These students are from two sections of high school and they are enrolled in the same course with the same professor. The first section carried out an activity of image formation using paper, pencil and ruler, while the other section did an activity of image formation through a computer simulation. The results showed that there was a significant advantage in the performance of the students who worked with, paper, pencil and ruler and that the computer-based teaching strategy may not always be the most beneficial for learning. (Benjelloun, Alami and Rebmann, 2004) evaluated, among students from the second year of university, the contribution of the use of Java Software of Geometrical Optics JSGO to overcome some conceptual difficulties in geometrical optics using a converging lens. These conceptual difficulties are related to the tracing of construction rays and its extensions when an optical component is moved; and they are also related to the physical sense attributed by the student to the formed optical image. These authors showed a "reverse evolution from correct answers with a correct justification using, paper and pencil to correct answers without justification by using JSGO”. 
In a second work, (Benjelloun, Alami and Rebmann, 2003) analyzed the integration of a Java Software of Geometrical Optics JSGO in the situations of solving a problem among thirty - one students in the second year of university, option: Sciences of Physical Matter SPM. Their results showed that, despite the high interactivity of JSGO with the optical components, the learners provided a false geometrical construction.

While researches have confirmed that the use of computers' technology is beneficial to teaching geometrical optics, others have shown that it is not easy to prove the positive influence of using such tool. From this point of view, we targeted through this work, an objective that lies in the study of the impact of using computer tool named "Java Software of Geometrical Optics JSGO" on the nature of geometrical construction of virtual image produced. And, in the case where this usage appears favorable, we will study the conceptual difficulty to which the integration of JSGO is useful.

The geometrical optics can explain and analyze some optical phenomena, such as the formation of images and the vision. However, a large part of geometrical optics cannot be assimilated without mastering the geometrical construction of optical image.

Mistrioti (2003) examined the tracing of construction ray extension for the vision of a virtual image among the students of CAPES and the DEUG. The results showed that the converging of construction rays extensions is less controlled than the tracing of one construction ray, which respects, according to the schemas analyzed, the refraction laws of Descartes. Also, the results indicated that only $31 \%$ of students presented in their schemas a construction ray extended in a straight line. (Galili and Hazan, 2000) presented models that illustrate the use of construction rays by the students during the study of light sources. They compared the use of construction ray according to the cactus model (scientifically correct) with its use according to the hybrid model (post-teaching) "Image Projection Conceptualization IPC".

According to the cactus model, the light source is a set of points and each of them distributes the luminous rays in all directions in space. However, according to the hybrid model IPC, the light source is considered by students as a set of points and each of them sends only one luminous ray carrying all the information of the source point. The analysis of students' knowledge facets, before and after teaching, revealed that a little students offer compatible explanations with the cactus model.Viennot (1996) has written: "Thus, a light ray is not a material object. It is a mode of representation, it is often called "model" used to express in a symbolic language the propagation of light. A luminous ray does not have the status of an object that is simply defined as a table for example. In particular, we cannot see the luminous rays and those that are believed to be seen, be they the "sun" or "laser", are in fact diffused particles that the light illuminates independently from one another. The "optical image" can be observed, but its rules of formation are surprising and are far removed from those governing the ordinary material objects".

In an investigation, Goldberg and McDermott (1987) studied the students' understanding of the images produced by converging lenses and concave mirrors, before and after teaching the geometrical optics. These authors showed that participants do not assimilate the unique character of the relation between the optical components: the function of the lens, the mirror and the screen. This study also showed the students' poor understanding of the tracing of construction rays and it extensions.

The research cited above enabled us to obtain an analysis, which is at the same time of epistemological (the status of construction ray), and didactic (the use of construction ray and it extension by students) and in order to contribute to the geometrical optic didactics, we have chosen to explore the "virtual" part of the formed optical image.

\section{Description of tests and learners' corpus}

\section{Research Framework}

During this research we developed within the framework of Gauss conditions two tests concerning the geometrical construction of the virtual image. Each of them consists of two variant sub-tests: A first variant subtests and a second variant sub-tests as described below:

The first variant sub-tests: 1-1;2-1 (Appendix 1) are simple and classical exercises of the construction of the virtual image we can find in the school textbooks.

The second variant sub-tests: 1-2; 2-2 (Appendix 1) are classical exercises of the geometrical construction of the virtual image which we have modified to render them less stereotyped and less unusual than the exercises studied often in the university.

Both tests are similar in content but they differ in the schemas. The sub-tests: 1-1 and 1-2 are respectively equivalent to the sub-tests: $2-1$ and 2-2. This equivalence has been proven by calculating the difficulty index of each sub-test among an external corpus of students who worked with the traditional method. The participants are from the first year of university, between 17 and 19 years old, option: Sciences of Physical Matter SPM. These participants have already received a classical teaching of the geometrical construction of a virtual image. 


\section{Description of Java Software of Geometrical Optics JSGO}

Java Software of Geometrical Optics that we denote by JSGO is a wizard that enables to realize interactive schemas and measurements of angles and distances (Rebmann, 2000). The window of JSGO (Figure 1) is constituted of a left part called "the store" that contains all the elements available, and of a right part reserved to the geometrical construction.

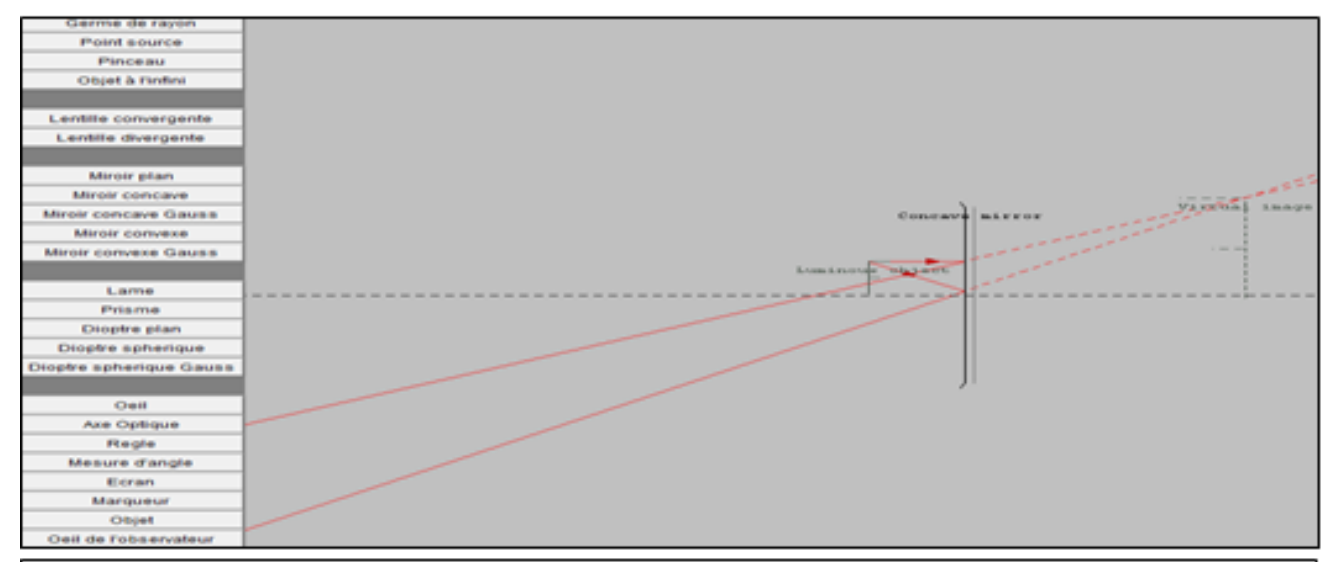

Figure 1: Geometrical construction of the image of an object placed between the principal focal object and the principal optical center of a concave mirror.

\section{Properties of JSGO}

The installation of Java software is essential to JSGO function, and the power of this software resides in it high degree of interactivity with the "optical components" and in particular, the tracing of the construction ray and its extension.

However, although the JSGO is sufficiently complete and efficient in offering a wide variety of objects with the possibility of acting on their properties, it has some limitations such as: the disappearance of the construction ray extension tracing when moving objects, also, the letters in the schema have a confusing nature. In addition, there is no possibility to save the geometrical constructions produced. The only means of keeping track is to make a "screen capture".

\section{Research questions}

The questions which our research is interested in are:

Does using JSGO represent a useful tool for the geometrical construction of the virtual image? What is (are) the conceptual difficulty (difficulties) for which the use of JSGO is helpful? What is the influence of using JSGO on the execution time-management?

\section{Research Methodology}

In answer to the research questions we have adopted a procedure based on:

- A comparative analysis between the successful number of geometrical construction produced by the witness group students and those related to the experimental group students.

- An analysis of the students' conceptual difficulties encountered in the false constructions produced.

- An analysis of the "students' perceptions about using JSGO" by using a Likert scale of 4 levels ranging from "strongly disagree" to "strongly agree".

\section{Taking place of experimentation}

The experimentation realized, took into account, the reality of the teaching in the university. It was conducted towards the end of the university year 2013/2014 among two groups of learners consisting each of them of 59 students: a witness group and another experimental.

The witness group students must to use only the traditional method, paper, pencil, ruler and protractor, for doing tests; while the experimental group students should construct the virtual image using JSGO.

Among the Guidelines that were given to all participants is that once tracing the construction rays and it extensions they must place the virtual images formed in their position. The time given to participants of the witness group students is 40 minutes (10 minutes for each sub-test); while the time given to the experimental group students is 60 minutes, in order to reproduce the schema initially provided (on paper) on the JSGO screen. 
Our decision to set a time limit is justified. Indeed the responses' time limit allowed us to study the impact of using JSGO on the execution time-management.

The experimental group participants have benefited from a formation of 45 minutes concerning the use of this tool. We have informed them that using JSGO does not permit to save the geometrical constructions produced, and that the only way to keep track is to make a "screen capture". We have showed them how to access the JSGO website; however we worked outside the context of online university, to avoid the risk of internet connection rupture. Each student of the experimental group worked individually on a computer.

\section{Analysis And Discussion Of Results}

1. Influence of the use of JSGO on the success of geometrical constructions produced.

The results of students' sub-tests are presented in table 1 below:

\begin{tabular}{ccccc}
\hline $\begin{array}{l}\text { Table 1: Number of correct constructions, false constructions and copies without constructions of students } \\
\text { for each sub-test }\end{array}$ \\
\cline { 3 - 5 } Sub-test & Group & CC & Nature of construction \\
\hline \multirow{2}{*}{ Sub-test 1-1 } & WG & 45 & FC & 13 \\
\hline \multirow{2}{*}{ Sub-test 1-2 } & EG & 46 & 11 & 2 \\
\hline \multirow{2}{*}{ Sub-test 2-1 } & WG & 25 & 30 & 4 \\
\hline \multirow{2}{*}{ Sub-test 2-2 } & EG & 51 & 8 & 2 \\
& WG & 43 & 14 & 3 \\
\hline
\end{tabular}

Codification of notations

$\mathbf{C C}=$ Correct Construction. $\mathbf{F C}=$ False Construction. $\mathbf{C W C}=$ Copy or "Screen capture" Without any Construction.

The experimental group students responded better than the witness group students. Indeed, the experimental group participants have respectively scored: $46,51,44$ and 47 correct constructions for the subtests: 1-1, 1-2, 2-1 and 2-2, against: 45, 25, 43 and 26 correct constructions produced by the witness group students.

Thereby we can conclude that the number of students who are able to produce correct constructions using JSGO is higher than the number of students who have produced correct constructions using the traditional method.

We noted a remarkable success of the number of geometrical construction produced by the witness group students for the first variant subtests compared with the second variant sub-tests. Indeed, these students scored respectively: 45 and 43 correct constructions for the sub-tests: 1-1 and 2-1, against: 25 and 26 correct constructions for the sub-tests: $1-2$ and 2-2.

This result shows that the presence of the opaque medium and the concave spherical diopter (refracting medium) in the respective schemas associated with the second variant sub-tests: 1-2 and 2-2 makes, for the witness group students, the construction of the virtual image more difficult than the construction of the virtual image when solving the first variant sub-tests: $1-1$ and 2-1.

\section{Analysis of the results using the Chi-square of Statistical Test}

To get an idea about the significant difference between the students' results related to the witness group students and those related to the experimental group students for each sub-test, we studied such difference using the Chi-square statistical test, called Test of independence Chi 2. The calculation of the Chi-square was carried using the predefined functions of Excel statistical software of Microsoft Office.

\section{Both hypotheses emitted to study the significant difference between the students' results}

Null hypothesis $\left(\mathbf{H}_{\mathbf{0}}\right)$ : "The student uses, or not, JSGO" and "the success, or not, of a sub-test by student" are independent.

Alternative hypothesis $\left(\mathbf{H}_{\mathbf{1}}\right)$ : "The student uses, or not, JSGO" and "the success, or not, of a sub-test by student" are not independent.

The results of Chi-square statistical Test are presented in table 2 below:

Table 2: The value of Chi-square, the interval of critical probability $p$ and the accepted hypothesis for each sub-test

\begin{tabular}{cccc}
\hline Sub-test & Chi-square value & Interval the critical probability: & The accepted hypothesis \\
\hline Sub- test 1-1 & 0,048 & $0,75<\mathrm{p}<0,9$ & $\mathrm{H}_{0}$ \\
Sub- test 1-2 & 24,989 & $\mathrm{p}<0,05$ & $\mathrm{H}_{1}$ \\
Sub- test 2-1 & 0,043 & $0,75<\mathrm{p}<0,9$ & $\mathrm{H}_{0}$ \\
Sub-test 2-2 & 15,841 & $\mathrm{p}<0,05$ & $\mathrm{H}_{1}$ \\
\hline
\end{tabular}


For the first variant sub-tests: $1-1$ and $2-1$, where the critical probability is more than $5 \%$, we cannot reject the null hypothesis $\left(\mathrm{H}_{0}\right)$ and we accept it. For the second variant subtests: 1-2 and 2-2, where the critical probability is less than $5 \%$, we can reject the null hypothesis $\left(\mathrm{H}_{0}\right)$ and accept the hypothesis $\left(\mathrm{H}_{1}\right)$. In other words, the results show no significant influence of using JSGO on the success of the first variant sub-tests: 1-1 and 2-1, although the use of this tool has a significant influence on the success of the second variant sub-tests: 12 and $2-2$.

Indeed, the non significant influence on the first variant sub-tests can be justified by the fact that, the schemas of the sub-tests: 1-1 and 2-1 is "poor" in "optical components" (just a plane mirror or concave spherical mirror). Therefore the power of JSGO which resides in its great interactivity with "optical components" becomes "low" and the construction of the virtual image using JSGO seems like a construction using traditional method.

The significant and positive impact of using JSGO on the success of the second variant sub-tests:1-2 and 2-2 can be justified by the fact that the schemas of these sub-tests are "rich" in "optical components". This takes advantage from the high interactivity of JSGO for the construction of the virtual image. The high interactivity of JSGO with the "optical components" associated with the schemas of these sub-tests facilitates the construction of the virtual image for the experimental group students, while the witness group students encounter some difficulty in the image construction using the traditional method.

According to the results presented in table 1, it can be concluded that using JSGO has a significant and positive influence on the geometrical constructions success in relation to the use of the classical method of construction when solving the second variant sub-tests.

\section{Students' conceptual difficulties}

We analyzed the geometrical constructions produced by classifying the false geometrical constructions according to the conceptual difficulties below:

CD. 1 = Conceptual difficulty related to the correspondence image object (punctual and global).

CD. 2 = Conceptual difficulty associated with the minimum number of rays departing from the same point of the luminous object.

CD. 3 = Conceptual difficulty related to the tracing of construction ray.

CD. 4 = Conceptual difficulty associated with the tracing of construction ray extension.

CD = Computer difficulty associated with the use of JSGO.

The results of the conceptual difficulties encountered by all students are presented in table 3 below:

\begin{tabular}{cccccccc}
\hline $\begin{array}{c}\text { Table 3: Number of conceptual difficulties experienced in the false constructions produced by students } \\
\text { for each sub-test }\end{array}$ \\
\cline { 3 - 8 } Sub-test & Group & \multicolumn{5}{c}{ Nature of difficulty } \\
\cline { 3 - 8 } & & CD.1 & CD.2 & CD.3 & CD.4 & CD \\
\hline \multirow{2}{*}{ Sub-test 1-1 } & WG & 6 & 4 & 5 & 5 & $* * * *$ \\
& EG & 5 & 5 & 0 & 0 & 1 \\
\hline \multirow{2}{*}{ Sub-test 1-2 } & WG & 4 & 5 & 3 & 20 & $* * * *$ \\
& EG & 5 & 5 & 0 & 0 & 0 \\
\hline \multirow{2}{*}{ Sub-test 2-1 } & WG & 5 & 4 & 6 & 5 & $* * * *$ \\
& EG & 6 & 6 & 0 & 0 & 0 \\
\hline \multirow{2}{*}{ Sub-test 2-2 } & WG & 7 & 4 & 4 & 18 & $* * * *$ \\
& EG & 6 & 3 & 0 & 0 & 1 \\
\hline
\end{tabular}

Before we start our discussion, we note that some false constructions have more than one conceptual difficulty.

For the first variant sub-tests: 1-1 and 2-1, the witness group students scored 5 difficulties related to the tracing of construction ray extension. This result shows that the geometrical construction associated with the first variant sub-tests do not allow the emergence of the conceptual difficulty related to the construction ray extension tracing. (About $9 \%$ of students encountered this conceptual difficulty).

For the second variant sub-tests: 1-2 and 2-2, the witness group students scored about 19 difficulties associated with the tracing of construction ray extension, against 0 difficulties for the experimental group students. Because of this, it can be concluded that the tracing of construction ray extension while solving the second variant sub-tests, presents a real difficulty for about $32 \%$ of the witness group students. The positive effect of using JSGO is expected because this software "traces" in an "automatic way" the construction ray extension. So, we can say that using JSGO represents a very useful tool to surmount this conceptual difficulty. However, it is difficult to know if we have really obtained a conceptual change associated with the tracing of construction ray extension. 
In connection with this conceptual difficulty, and in the false schemas corresponding to the sub-test 12 , about $34 \%$ of witness group students consider that the opaque medium "prevents" the formation of the virtual image (Figure 10, Appendix 2). In this respect we can say that about $34 \%$ of the witness group students consider that the opaque medium "blocks" the construction ray extension. In the false schemas corresponding to the sub-test 2-2, about $30 \%$ of the witness group students consider that the spherical concave diopter has an "effect" on the formation of the image (Figure 11, Appendix 2). About $30 \%$ of these students consider that the construction ray extension "undergoes" refraction in relation to the axis of construction ray, modeling, the luminous ray reflected on the spherical concave mirror.

For all sub-tests, the number of the difficulties associated with the correspondence image object (punctual and global) proven in the false geometrical constructions produced by the experimental group students is about 6 (about $10 \%$ of students encountered this conceptual difficulty for each sub-test).

For all sub-tests, the experimental group students produced about 5 difficulties associated with the minimum number of rays departing from the same point of the luminous object, and also related to the correspondence image-object (punctual and global). This result illustrates that about $9 \%$ of these students encountered this conceptual difficulty. In the false geometrical constructions corresponding to this conceptual difficulty, we note that one construction ray departing from a point of the luminous object has been traced, or a lot of construction rays have been traced, but each one departs from a different point of the luminous object. This result reflects that despite the high interactive character of the JSGO, $9 \%$ of the experimental group students come up against this conceptual difficulty.

The analysis showed that using JSGO generates a little difficulty. Indeed, approximately 2 difficulties have been recorded in the "screen captures" corresponding to these false constructions. In fact, we noticed a "poor" reproduction of the schema on JSGO screen (The schema on "screen capture" does not reflect the schema corresponding to the sub-test submitted).

\section{Students' perceptions about using JSGO}

To analyze the students' perceptions we used a Likert scale of 4 levels ranging from "strongly disagree" to "strongly agree".

Level 1: "Strongly disagree" assigned to the weighted value 1

Level 2: "Disagree" assigned to the weighted value 2.

Level 3: "Agree" assigned to the weighted value 3.

Level 4: "Strongly agree" assigned to the weighted value 4.

The results of students' perceptions are presented in table 4 below:

Table 4: Grid of learner' perceptions concerning the use of JSGO

\begin{tabular}{|c|c|c|c|c|c|}
\hline The proposed affirmation & $\begin{array}{l}\text { Strongly } \\
\text { disagree }\end{array}$ & Disagree & Agree & $\begin{array}{c}\begin{array}{c}\text { Strongly } \\
\text { agree }\end{array} \\
\end{array}$ & $\begin{array}{c}\text { The average } \\
\text { ranking }\end{array}$ \\
\hline The menu of JSGO is clear & $\begin{array}{c}\mathbf{0} \\
\mathbf{0 \%} \\
\end{array}$ & $\begin{array}{c}3 \\
5 \%\end{array}$ & $\begin{array}{c}8 \\
14 \%\end{array}$ & $\begin{array}{c}48 \\
81 \%\end{array}$ & 3,76 \\
\hline The JSGO is well adapted to my needs & $\begin{array}{c}4 \\
7 \%\end{array}$ & $\begin{array}{c}6 \\
10 \%\end{array}$ & $\begin{array}{c}4 \\
7 \%\end{array}$ & $\begin{array}{c}45 \\
76 \%\end{array}$ & $\mathbf{3 , 5 2}$ \\
\hline $\begin{array}{l}\text { The use of JSGO is motivating as a traditional method of } \\
\text { construction }\end{array}$ & $\begin{array}{c}1 \\
1 \%\end{array}$ & $\begin{array}{c}8 \\
14 \% \\
\end{array}$ & $\begin{array}{c}10 \\
17 \% \\
\end{array}$ & $\begin{array}{c}40 \\
68 \% \\
\end{array}$ & 3,50 \\
\hline The JSGO assisted me to construct the virtual image & $\begin{array}{c}2 \\
3 \%\end{array}$ & $\begin{array}{c}7 \\
12 \%\end{array}$ & $\begin{array}{c}11 \\
19 \%\end{array}$ & $\begin{array}{c}39 \\
66 \%\end{array}$ & $\mathbf{3 , 4 7}$ \\
\hline I can manage my time using JSGO & $\begin{array}{c}6 \\
10 \%\end{array}$ & $\begin{array}{c}\mathbf{5} \\
\mathbf{8 \%} \\
\end{array}$ & $\begin{array}{c}13 \\
22 \% \\
\end{array}$ & $\begin{array}{c}35 \\
60 \% \\
\end{array}$ & $\mathbf{3 , 3 0}$ \\
\hline
\end{tabular}

Note: The number in each cell represents the students' numbers who have chosen a particular level for the proposed affirmation.

Generally, the grid of learners' perceptions indicates that the majority of students are agree with the proposed affirmations concerning the use of JSGO. Regarding the proposition "the use of JSGO assisted me to construct the virtual image" the average of 3,47 means that the result is found nearer to the level "agree". This can be explained by the important interactivity offered by JSGO. Likewise, $81 \%$ of participants chose the level "strongly agree" to the proposition "the menu of JSGO is clear". For the proposition associated with the time management, $18 \%$ of the learners are unable to manage their time using JSGO. However, the average of 3,30 indicates that the result of students' perceptions is found nearer to the level "agree". The use of this software rendered the geometrical construction of the virtual image more motivating and attractive than a construction using a traditional method. This is proven by the average of 3,50 concerning the proposition "the use of JSGO is motivating as a traditional method of construction". 


\section{Conclusion}

This work has enabled us to study the impact of the use of Java Software of Geometrical Optics JSGO on the success of the geometrical construction of the virtual image among the first university students, option: Sciences of Physical Matter SPM

By analyzing the difficulties proven in the false geometrical constructions produced by the witness group students, we have found that the first variant sub-tests do not allow the emergence of the conceptual difficulty associated with the tracing of construction ray extension.

The geometrical construction of the virtual image through the second variant sub-tests has shown that about $32 \%$ of witness group students have a misconception associated with the role of the construction ray extension. This result joined that of (Mistrioti, 2003) concerning the emergence of this conceptual difficulty, even for university students, by working with an optical system formed of a plane diopter.

We have scored that using JSGO has no significant influence on the success of the geometrical construction of the virtual image associated with the first variant sub-tests. However, using this tool through the second variant sub-tests leads to a number of correct constructions which is higher than that noticed when using traditional method. This expected result raised some questions about the importance of the introduction of adequate pedagogical scenarios that help get a good profit from this computer-assistant learning.

It is true that the technique of tracing of construction ray and it extension using JSGO can be performed in an algorithmic way, without necessarily mastering this technique by the students. In fact, it is not easy to know if the improvement of the results is really due to a better understanding of knowledge brought into play when solving the second variant sub-tests. However, using JSGO permits to overcome this conceptual difficulty. We scored a difference between our results associated with the sub-test 1-2 and those of Mistrioti (2003) concerning the schema given to the students to complete. Indeed, $34 \%$ of the witness group students have provided a correct construction for the sub-test $1-2$, while just $11 \%$ of multidisciplinary students scored a correct construction. This difference can be justified by the fact that the mechanism of the vision of an image is more difficult than a simple geometrical construction of the virtual image. In fact, the study of the mechanism of the vision of a virtual image involves a "double geometrical construction" The geometrical construction of the optical image of the luminous object through the optical system, and the geometrical construction of the image of the formed optical image through the "eye" that constitutes another independent optical system.

This analysis also indicates that, despite the interactive character of JSGO, about $9 \%$ of students face the conceptual difficulty associated with the correspondence image object (punctual and global), and also the difficulty related to the minimum number of construction rays departing from the same point of the luminous object. This result joined that of (Benjelloun and al, 2003) concerning the production of false geometrical constructions by the students in the second years of university even using JSGO.

Finally, we note that our study "students' perceptions about using JSGO" showed that the participants are very satisfied regarding the use of this computer wizard.

\section{References}

[1]. Ahaji, K., El Hajjami., A., Ajana, L., El Mokri, A., \& Chikhaoui, A. (2008). Analyse de l'effet d'intégration d'un logiciel d'optique géométrique sur l'apprentissage d'élèves de niveau baccalauréat sciences expérimentales». Association Enseignement Public et Informatique. Available on the website: http://www.epi.asso.fr/revue/articles/a0801a.htm

[2]. Bayrak, C. (2008). Effects of Computer Simulations Programs on University Students' Achievements in Physics. Turkish online Journal of Distance Education, vol. 9, $\mathrm{n}^{\circ} 4$, p. 53-62.

[3]. Benjelloun, N., Alami, M., \& Rebmann, G. (2003). Expérimentation d'un atelier java d'optique géométrique (AJOG) en situation de résolution de problème. Bulletin de l'union des physiciens, vol. 97, p. 1613-1621. Available on the website: http://agregation.capes.free.fr/bup/sommaires-2003bup.htm

[4]. Benjelloun, N., Alami, M., \& Rebmann, G. (2004). Evaluation de l'utilisation d'un atelier java d'optique géométrique (AJOG) pour remédiation à certaines difficultés conceptuelles en optique. Communication présentée au $21^{\text {ème }}$ Congrès de l'Association Internationale de Pédagogie Universitaire AIPU. p. 1-11. Cadi Ayyad University, Marrakech, Morocco.

[5]. Clark, J. (2011). Technology in the classroom: student understanding of image formation by converging and diverging lenses and mirrors-ray tracing. Computer simulation (Doctoral dissertation, ball state university).

[6]. Galili, I., \& Hazan A. (2000). Learner's Knowledge in Optics: Interpretation, Structure and Analysis. International Journal of Science Education, vol. 22, n⿳⺈ 1, p. 57-88.

[7]. Goldberg, F.M., \& Mc Dermott, L.C. (1987). An investigation of student understanding of the real image formed by a converging lens or concave mirror. American Journal of Physics. Vol. 55, $\mathrm{n}^{\circ}$ 2, p. 108-119. Available on the website: http://physics.ucsd.edu/students/courses/winter2006/managed/physics180_280/documents/goldbergmcd87.pdf

[8]. Mistrioti, Y. (2003). Optique géométrique et interprétation de la vision par les étudiants universitaires: un modèle d'interprétation de la vision d'une image virtuelle. (Thèse de doctorat non disponible en ligne). Université Denis Diderot Paris 7, Paris, France.

[9]. Rebmann, G. (2000). Atelier java d'optique géométrique [Software]. Available on the website :http://www.uelpcsm.education.fr/consultation/presentation/present/maquette/exemples_uel/module_ex/simuler/phy/ex3/atelier.htm Viennot, L. (1996). Raisonner en physique - la part du sens commun. Paris: De Boeck University. 


\section{Test 1:}

\section{$\underline{\text { Appendix } 1}$}

\section{Sub-test 1-1}

Enunciated: A luminous object of small dimensions in the form of the letter F, is placed vertically in vacuum (the index of refraction of vacuum is $n=1$ ) in front of a plane mirror (PM) (Figure 2). Construct, geometrically, the image $F_{1}$ of the luminous object $F$ through the plane mirror (PM).

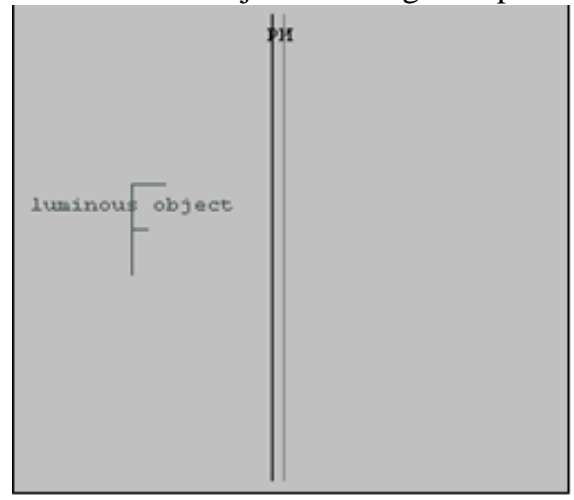

Figure 2: schema of sub-test 1-1

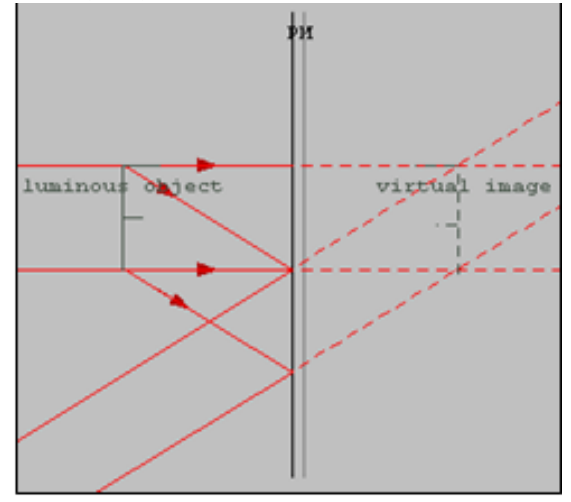

Figure 3: correct construction of sub-test 1-1

Sub-test 1-2

Enunciated: Now, we place an opaque medium tangent on the right side of the plane mirror (PM) (Figure 4). Construct geometrically, the image $\mathrm{F}_{2}$ of the luminous object $\mathrm{F}$ through the system: $\{(\mathrm{PM})+$ opaque medium $\}$.

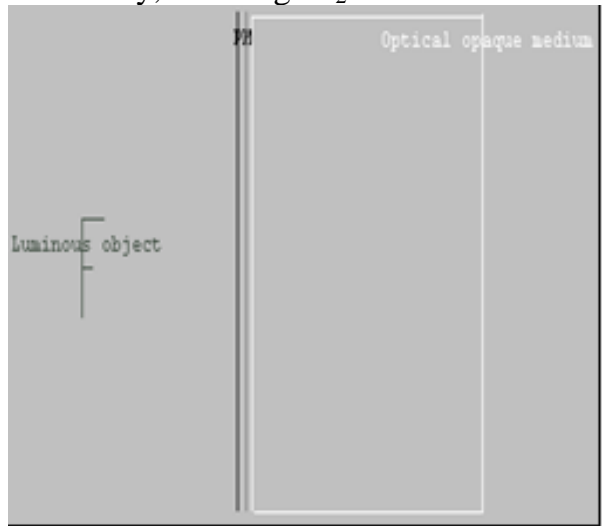

Figure 4: schema of sub-test 1-2

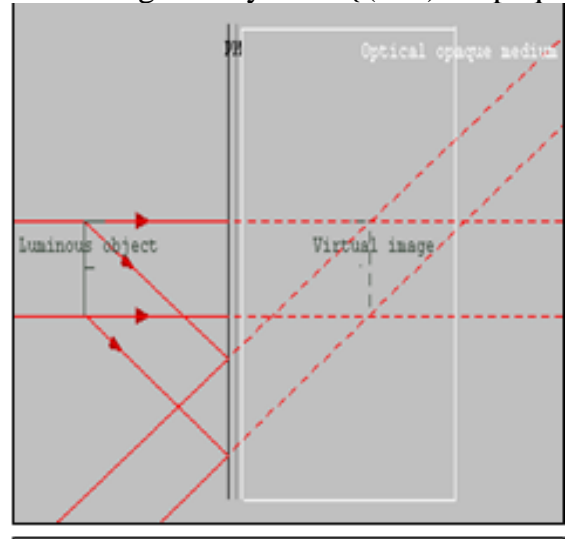

Figure 5: correct construction of sub-test 1-2

\section{Test 2:}

Sub-test 2-1

Enunciated: A luminous object of small dimensions in the form of the letter F, is placed vertically in vacuum (the index of refraction of vacuum is $n=1$ ) in front of a spherical concave mirror (CM) (Figure 6). Construct, geometrically, the image $\mathrm{F}_{3}$ of the luminous object $\mathrm{F}$ through the spherical concave mirror $(\mathrm{CM})$. 


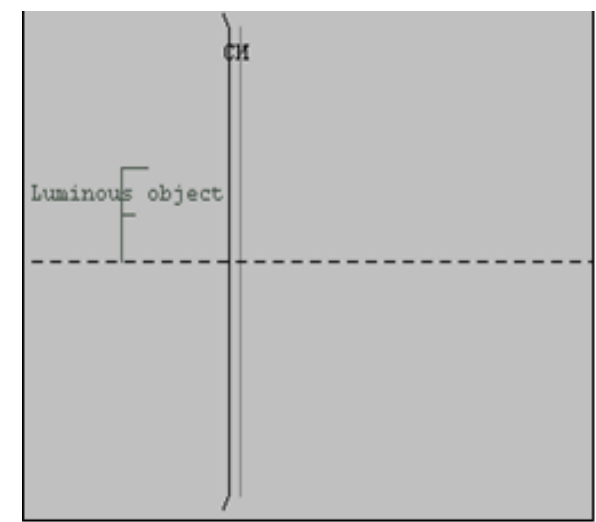

Figure 6: Schema of sub-test 2-1

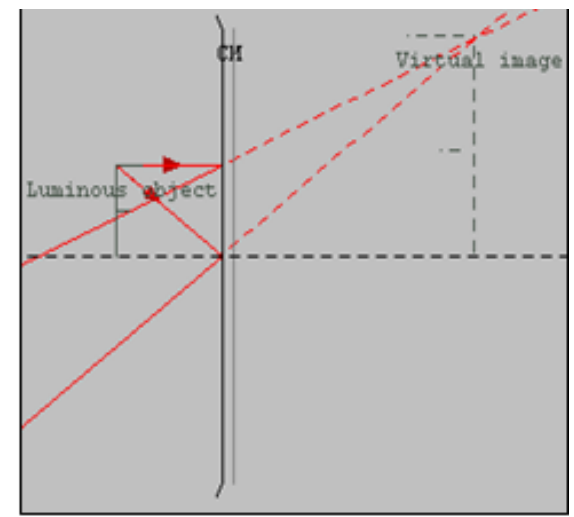

Figure 7: correct construction of sub-test 2-1.

\section{Sub-test 2-2}

Enunciated: Now, we place a refracting medium (the refraction index of this medium is $n=1,5$ ) tangent on the right side of spherical concave mirror (Figure 8). Construct geometrically $\mathrm{F}_{4}$ the image of the luminous object $\mathrm{F}$ through the system: $\{(\mathrm{CM})+$ refracting medium $\}$.

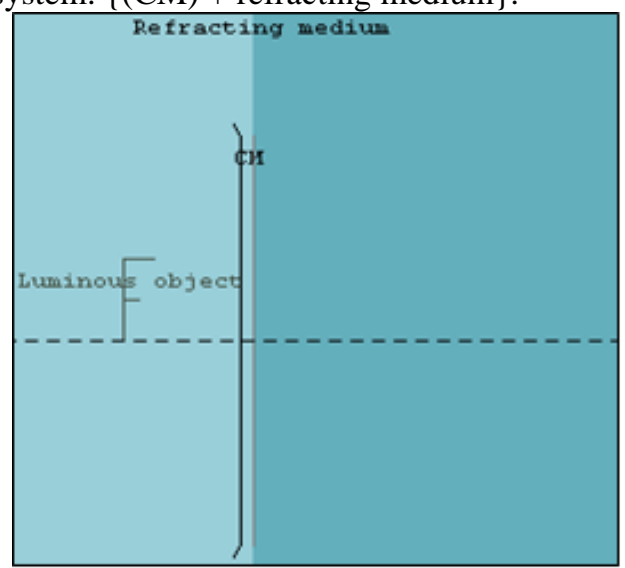

Figure 8: Schema of sub-test 2-2

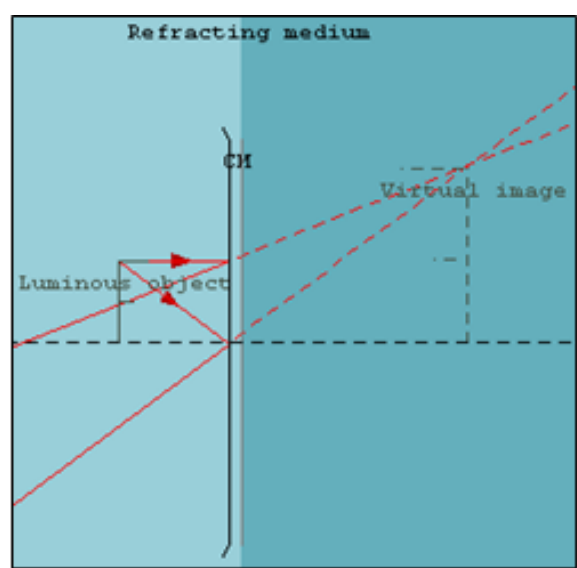

Figure 9: correct construction of sub-test 2-2

Appendix 2

False constructions associated with the sub-tests: 1-2 and 2-2.

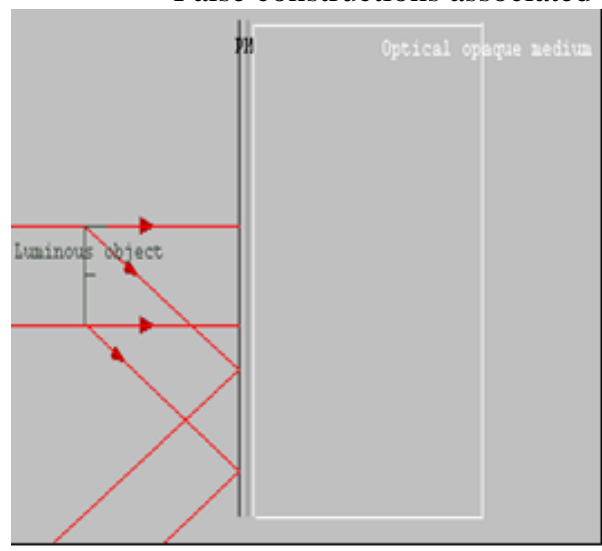

Figure 10: A false construction in which the construction rays extensions are "blocked" by the opaque medium.

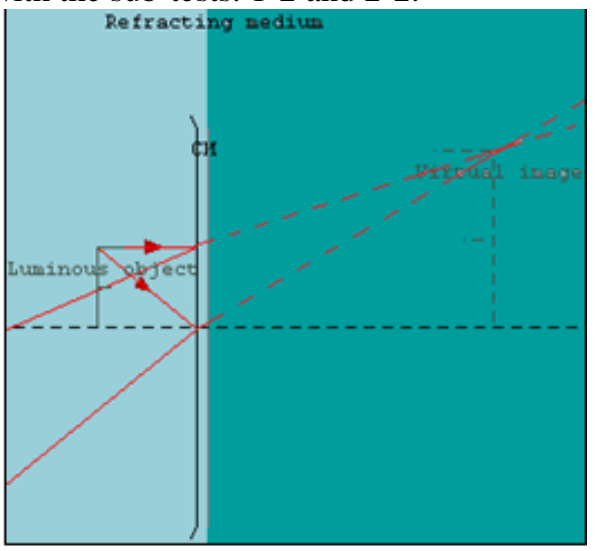

Figure 11: A false construction in which the construction rays extensions have "undergo" refraction by the refracting medium. 